\title{
Association Between Retinal Sensitivity and the Presence of Quiescent Choroidal Neovascularization in Pachychoroid Diseases
}

\section{Rion Ozawa}

Department of Ophthalmology, Graduate School of Medicine and Faculty of Medicine, The University of Tokyo, Tokyo 113-8655, Japan

Ryo Obata ( $\nabla$ robata-tky@umin.ac.jp )

Department of Ophthalmology, Graduate School of Medicine and Faculty of Medicine, The University of Tokyo, Tokyo 113-8655, Japan

\section{Keiko Azuma}

Department of Ophthalmology, Graduate School of Medicine and Faculty of Medicine, The University of Tokyo, Tokyo 113-8655, Japan

\section{Yoko Nomura}

Department of Ophthalmology, Graduate School of Medicine and Faculty of Medicine, The University of Tokyo, Tokyo 113-8655, Japan

\section{Hiroshi Murata}

Department of Ophthalmology, Graduate School of Medicine and Faculty of Medicine, The University of Tokyo, Tokyo 113-8655, Japan

\section{Ryo Asaoka}

Department of Ophthalmology, Seirei Hamamatsu General Hospital, Shizuoka, Japan. Seirei Christopher University, Shizuoka, Japan

\section{Kohdai Kitamoto}

Department of Ophthalmology, Graduate School of Medicine and Faculty of Medicine, The University of Tokyo, Tokyo 113-8655, Japan

\section{Kohei Ueda}

Department of Ophthalmology, Graduate School of Medicine and Faculty of Medicine, The University of Tokyo, Tokyo 113-8655, Japan

\section{Tatsuya Inoue}

Department of Ophthalmology and Micro-Technology, Yokohama City University School of Medicine, 457 Urafune-cho, Minami-ku, Yokohama, Kanagawa, 232-0024, Japan

\section{Research Article}


Keywords: Central Serous Chorioretinopathy, Choroidal vascular hyper permeability, Retinal sensitivity, Quiescent choroidal neovascularization, Pachychoroid diseases

Posted Date: September 22nd, 2021

DOl: https://doi.org/10.21203/rs.3.rs-910002/v1

License: (c) (1) This work is licensed under a Creative Commons Attribution 4.0 International License. Read Full License 


\section{Abstract}

This study was conducted to examine retinal sensitivity (RS) in eyes with pachychoroid diseases and to analyze its association with the presence or absence of quiescent choroidal neovascularization (CNV), that can be protective against retinal dysfunction or atrophy in other macular diseases such as agerelated macular degeneration. A total of 12 eyes of 12 patients aged $\geq 45$ years having the characteristic findings of central serous chorioretinopathy but not presenting any exudative changes were included in this study. Choroidal vascular hyper permeability $(\mathrm{CVH})$ was identified by indocyanine green angiography, and the presence or absence of CNV was evaluated by optical coherence tomography angiography. RS at 68 points was examined by microperimetry. The average RS corresponding to within and outside $\mathrm{CVH}$ was compared. The association between the difference in RS and the presence or absence of CNV was also analyzed. CNV was detected in six eyes (50\%). In eyes without CNV, the RS within CVH was similar compared with that outside CVH. However, in eyes with CNV, the RS within CVH was significantly decreased compared with that outside $\mathrm{CVH}$. Multiple regression analysis revealed the presence of $\mathrm{CNV}$ as an independent factor associated with RS. In eyes with pachychoroid diseases, RS decreased within the $\mathrm{CVH}$ area under the coexistence of nonexudative CNV.

\section{Introduction}

Pachychoroid diseases are a series of clinical entities that share some characteristic findings in the retina or the choroid ${ }^{1-3}$. The most important finding is a focally or diffusely thickened choroid that contains dilated choroidal vessels and attenuated choriocapillaris accompanied with abnormalities of the retinal pigment epithelium (RPE) ${ }^{1}$. The lesion is often characterized by choroidal vascular hyper permeability $(\mathrm{CVH})$ revealed by indocyanine green angiography (ICGA). The clinical entities include pachychoroid pigment epitheliopathy (PPE) ${ }^{4,5}$, chronic central serous chorioretinopathy (CSC), pachychoroid neovasculopathy 6,7 , polypoidal choroidal vasculopathy $(\mathrm{PCV})^{6,7}$, and pachychoroid geographic atrophy 8-11. During the course of these diseases, they present exudative changes such as serous detachment of the retina or the RPE with or without choroidal neovascularization (CNV) and atrophic changes at the choriocapillaris, the RPE, or the sensory retina. The area of $\mathrm{CVH}$ has been reported to be anatomically linked with these changes ${ }^{12,13}$. To date, increasing evidence has suggested that pachychoroid diseases should be distinguished from age-related macular degeneration (AMD), which exhibits similar exudative or atrophic changes in the posterior retina ${ }^{14}$.

Recent advancement of imaging technology, especially that of optical coherence tomography angiography (OCTA), has contributed to increased detection of CNV or macular neovascularization (MNV), especially quiescent or nonexudative CNV or nonexudative MNV, i.e., neovascular tissue not accompanied with exudative changes. In a previous review, nonexudative MNV was detected in $6-27 \%$ of the fellow eyes with AMD ${ }^{15}$. Nonexudative MNV could be a precursor for exudative AMD, but it may retard the growth of geographic atrophy and could have a protective effect on retinal function, probably at least in the stage where the lesion was quiescent ${ }^{15,16}$. 
Meanwhile, several studies have reported the presence of nonexudative CNV in pachychoroid diseases 17,18. The presence of nonexudative CNV could also be a risk factor for the development of exudative changes in CSC $^{19}$ and PCV ${ }^{20}$. However, whether nonexudative CNV affects the retinal function is not clearly understood.

A previous study ${ }^{21}$ showed that eyes with chronic CSC complicated with CNV demonstrated worse visual or reading acuity than those without CNV. However, considering that the lesion does not necessarily involve the fovea, it is important to not only compare visual acuity but also analyze retinal sensitivity (RS) at the lesion area. Nevertheless, to the best of our knowledge, there has been no published report investigating the association between RS and the presence or absence of quiescent CNV.

Therefore, in the present study, we investigated RS by microperimetry in eyes with pachychoroid diseases. We then evaluated the local function corresponding to within or outside the lesion area. In addition, we examined the association between the presence or absence of CNV and the changes in RS.

\section{Methods}

This retrospective study was conducted according to the tenets of the Declaration of Helsinki and was approved by the institutional review board (IRB) of the University of Tokyo. Written informed consent was not required by the IRB, but participants who did not grant authorization to use their medical records for the research were excluded from the study.

\section{Participants}

We retrospectively reviewed the medical charts of consecutive patients, including 12 eyes of 12 patients with PPE who visited the University of Tokyo Hospital from April 2018 to July 2019. All subjects met the following criteria: (1) patients were diagnosed with CSC with CVH lesions in the examined eye, and (2) patients had not shown any exudative changes or hemorrhage for more than 6 months by the time of RS examination. Patients who were treated with photodynamic therapy or antivascular endothelial growth factor therapy were not included. Patients complicated with other retinal diseases such as diabetic retinopathy, retinal vascular diseases, myopic maculopathy, glaucoma, and significant cataract that could affect visual function were also excluded.

\section{Multimodal imaging}

All patients were examined by fluorescein angiography and ICGA (Heidelberg Retina Angiograph 2; Heidelberg Engineering, Heidelberg, Germany), spectral-domain optical coherence tomography (OCT) (Heidelberg Engineering $\mathrm{GmbH}$, Dossenheim, Germany), and OCTA for diagnosis. Central choroidal thickness (CCT) was also measured on OCT based on enhanced depth images.

\section{OCTA measurement}


OCTA images were acquired using the Angiovue software of RTVue XR Avanti (Optovue, Inc., Fremont, CA). OCTA was performed using the split-spectrum amplitude-decorrelation angiography system. OCTA images were obtained with a $3 \times 3 \mathrm{~mm}$ and $6 \times 6 \mathrm{~mm}$ macular cube centered on the lesion, and an automated segmentation layer was set to both the outer retina and the choriocapillaris. Then, we manually moved the slab to detect the neovascular lesions, referring to the B-mode scan ${ }^{23}$. Two independent investigators (KA and RO) evaluated the images for the presence of CNV.

\section{Identification of $\mathrm{CVH}$}

CVH was evaluated using ICGA images (Fig. 1a, 1b). On ICGA, CVH was defined as irregular areas of increased fluorescence during the mid and late phases, often surrounding dilated pachyvessels ${ }^{24}$. Identification of the $\mathrm{CVH}$ area was conducted by two experienced retinal specialists (KA and YN) using the ICGA image in the mid- and late-phase study (Fig. 1c) 24,25 .

\section{0 - 2 VF measurement}

Each patient underwent VF testing with the AP-7000 automatic perimeter (KOWA Company Ltd., Tokyo, Japan) using a $10^{\circ}$ radius from the fovea. A white-on-white $10-2$ measurement was conducted using the Swedish interactive threshold algorithm standard test and the standard Goldmann size III stimulus and tested at 68 points. After the completion of the sensitivity testing, the sensitivity values were superimposed on the corresponding site in the ICGA image using the built-in software (Fig. 1d). The difference in average sensitivities between the inside and outside of $\mathrm{CVH}$ was calculated in each eye ( $\Delta$ Retinal Sensitivity o-i; $\triangle$ RSo-i).

\section{Statistical analysis}

Demographic characteristics (age, sex, CCT, and BCVA) were compared between subjects with and without CNV using the chi-square test for categorical variables and the paired $t$-test for continuous variables. Mean RS within or outside $\mathrm{CVH}$ was calculated in the total, $\mathrm{CVH}+$, or $\mathrm{CVH}-$ eyes. Within the eyes with CNV or those without CNV, $\triangle \mathrm{RS}$-i was analyzed using the Mann-Whitney U test. The mean $\triangle \mathrm{RSO}-\mathrm{i}$ values were also compared between both groups using the Mann-Whitney $U$ test. The relationship between $\triangle \mathrm{RSO}$-i and age, sex, CCT, or the presence or absence of CNV was analyzed by univariate and multivariate analyses. In univariate analysis, linear regression models were used for continuous variables, whereas logistic regression models were used for binary outcomes. Multiple explanatory variables were analyzed by multiple regression analysis, followed by model selection using the second-order bias-corrected Akaike information criterion. Hence, the optimal linear model was then selected among all possible combinations of predictors $\left(2^{4}\right.$ patterns when there were four explanatory variables). The variables included in the optimal model were considered significant. P-values less than 0.05 were considered statistically significant.

All statistical analyses were conducted using the JMP version 11.0 software program (SAS Institute, Cary, NC, USA).

Results 
In total, 12 eyes of 12 patients were included. The mean age of the patients was $52.3 \pm 9.4$ years (mean \pm standard deviation: SD, range: 47-69 years), and there were nine male patients. The characteristics of the patients are shown in Table 1. CNV was detected by OCTA in six eyes (50\%).

Table 1

Demographic characteristics of the patients

\begin{tabular}{|lllll|}
\hline & Total & CNV+ & CNV- & p value \\
\hline Case (eyes) & 12 & 6 & 6 & \\
\hline Age (mean \pm SD) [range] & $\begin{array}{l}52.3 \pm 9.4 \\
{[47-69]}\end{array}$ & $50.0 \pm 11.2[47-67]$ & $\begin{array}{l}54.7 \pm 7.7 \\
{[47-69]}\end{array}$ & $0.42^{*}$ \\
\hline Male sex (patients) & 9 & 4 & 5 & $0.51^{\dagger}$ \\
\hline CCT $(\mu \mathrm{m})$ & $351 \pm 127$ & $387 \pm 110$ & $315 \pm 142$ & $0.35^{*}$ \\
\hline BCVA (logMAR) & $0.02 \pm 0.12$ & $0.002 \pm 0.02$ & $0.018 \pm 0.14$ & $0.67^{*}$ \\
\hline *Mann-Whitney U test; ${ }^{+}$Chi-square test & & & \\
\hline
\end{tabular}

Variables were compared between eyes with and without CNV. There was no statistical difference in age, sex, or CCT. The RS outside CVH was similar between CNV + and CNV - eyes, whereas the RS inside CVH was numerically lower in CNV + eyes than in CNV - eyes. RS inside CVH was significantly lower than that outside CVH in the CNV + group $(-1.60 \pm 1.51, p=0.016)$ but not in the CNV - group $(-0.04 \pm 0.68, p=$ 0.64 , Table 2). $\triangle$ RSo-i was significantly better in eyes without CNV than in eyes with CNV eyes $(p=0.047$, Fig. 2).

Table 2

The difference in average sensitivities between the inside and outside of $\mathrm{CVH}(\triangle \mathrm{RSo}-\mathrm{i})$ in eyes with $\mathrm{CNV}$ $(\mathrm{CNV}+, \mathrm{n}=6)$ or without CNV $(\mathrm{CNV}-, \mathrm{n}=6)$

\begin{tabular}{|lllll|}
\hline & Total & CNV+ & CNV- & P value* \\
\hline RS outside CVH & $-1.44 \pm 1.12$ & $-1.37 \pm 0.99$ & $-1.50 \pm 1.34$ & 0.17 \\
\hline RS inside CVH & $-2.25 \pm 1.82$ & $-2.95 \pm 2.07$ & $-1.54 \pm 1.34$ & 0.016 \\
\hline$\triangle$ RSo-i $(\mathrm{dB})$ & $-0.81 \pm 1.38$ & $-1.60 \pm 1.51$ & $-0.04 \pm 0.68$ & 0.047 \\
P value $^{\ddagger}$ & & 0.016 & 0.64 & \\
\hline
\end{tabular}

*Mann-Whitney $\mathrm{U}$ test between groups. ${ }^{\ddagger}$ Mann-Whitney $\mathrm{U}$ test within the eyes of each group. Statistical difference from zero was analyzed.

\section{Association between $\triangle \mathrm{RSO}-\mathrm{i}$ and demographic or anatomic factors}


In multivariate analysis, the presence of CNV was included in the optimal model for $\triangle$ RSo-i. Specifically, eyes with CNV were associated with the decrease in RS (Table 3).

Table 3

Results of the optical model selection for $\triangle \mathrm{RSO}-\mathrm{i}$

\begin{tabular}{|llllll|}
\hline & $\begin{array}{l}\text { Univariate } \\
\text { Analysis }\end{array}$ & & $\begin{array}{l}\text { Multivariate } \\
\text { Analysis }\end{array}$ & & \\
\hline Parameters & coefficient & p value & coefficient & standard error & p value \\
\hline Age (years) & 52.3 & 0.20 & N.S. & & \\
\hline Sex & 3.01 & 0.22 & N.S. & & \\
CCT $(\mu \mathrm{m})$ & 351.2 & 0.48 & N.S. & & \\
\hline CNV absent/present & 7.1 & 0.046 & 0.77 & 0.34 & 0.047 \\
\hline
\end{tabular}

\section{Discussion}

This study was conducted to analyze the association between $\mathrm{CVH}$ and RS in eyes with pachychoroid diseases, focusing on the presence of CNV. Results showed that the presence of CNV was associated with the decrease in RS.

We observed that the RS within and outside $\mathrm{CVH}(\triangle \mathrm{RSo}-\mathrm{i})$ was significantly different only in eyes with $\mathrm{CNV}$. Furthermore, univariate and multivariate analyses revealed that the presence of CNV was an independent factor associated with $\triangle \mathrm{RSO}$-i. Studies have reported that in pachychoroid diseases, the area of $\mathrm{CVH}$ is anatomically linked with atrophy or CNV ${ }^{12,13}$. However, there is no clear understanding of the changes in retinal function in $\mathrm{CVH}$ and the association with the presence or absence of CNV.

Sulzbacher et al. divided chronic CSC into neovascular CSC and nonneovascular CSC ${ }^{21}$. They found that CNV was detected in $34.5 \%$ of eyes with chronic CSC by OCTA. Their study showed that eyes with CSC demonstrated worse distance visual acuity at the initial or final visit and worse reading acuity at the final visit in the presence of $\mathrm{CNV}{ }^{21}$. These results indicate that retinal function could be worsened, especially with CNV. However, considering that the lesion does not necessarily involve the fovea, it is essential to not only compare visual acuity but also analyze the local function within or outside the lesion to examine functional changes. Therefore, we evaluated the RS within or outside the lesion and found that it was significantly decreased inside CVH but only in eyes with nonexudative CNV. The mechanism underlying the decrease in retinal function in $\mathrm{CVH}$ in eyes with $\mathrm{CNV}$ remains unclear. Considering that $\mathrm{CNV}$ generally develops within the $\mathrm{CVH}$ area, there are two hypotheses: one is that persisting detrimental process in the RPE or the sensory retina induced by CNV, although it appears quiescent, causes progressive retinal atrophy. The other hypothesis is that the CVH that could cause CNV should be more ischemic or should have more aberrant circular condition, which could result in atrophic changes in the retina and the RPE. However, our study could not address this point because of its retrospective design. A further prospective 
study may elucidate a more detailed course of functional changes or the cause-and-effect relationship of the functional changes in the retina and the development of CNV.

In eyes without CNV, RS were comparable in terms of between the inside and outside of $\mathrm{CVH}$. To the best of our knowledge, there have been no reports investigating the RS changes in $\mathrm{CVH}$. The presence of $\mathrm{CVH}$ suggests vascular congestion and choriocapillaris damage, leading to relative choroidal ischemia ${ }^{1}$. The changes are more common in eyes with CSC and PPE than in eyes with uncomplicated pachychoroid diseases ${ }^{22}$. Hence, $\mathrm{CVH}$ could indicate a pathological condition that predisposes to CSC or PPE. However, the results of the present study suggest that $\mathrm{CVH}$ per se may not lead to retinal dysfunction.

There were several limitations in our study. First, the sample size was small. Second, it was a retrospective study, which may have led to selection bias. Furthermore, although we included only patients with quiescent CNV, i.e., CNV that was not accompanied with exudative changes for more than 6 months, the possibility that serous detachment due to CSC or serosanguinous changes due to CNV previously exist and cause irreversible retinal dysfunction cannot be excluded. This potential selection bias could not be discussed because of our study design. However, because these changes generally evolve irrespective of the $\mathrm{CVH}$ lesion, the RS changes within $\mathrm{CVH}$ could not be explained completely by these exudative changes but, at least in part, by the atrophic changes in $\mathrm{CVH}$ per se. We believe that the presence or absence of nonexudative $\mathrm{CNV}$ in $\mathrm{CVH}$ would provide more information.

\section{Conclusions}

The RS within CVH was decreased in patients with nonexudative CNV. Detecting quiescent CNV by OCTA may be helpful in suspecting retinal dysfunction in CVH in patients with pachychoroid diseases.

\section{Declarations}

\section{Ethics approval and consent to participate}

The study was conducted in accordance with the tenets of the Declaration of

Helsinki and with the approval of the ethics committee at the coordinating center of the University of Tokyo. All patients provided written informed consent prior to participation in the study.

\section{Consent for publication}

Not applicable.

\section{Availability of data and materials}


The datasets generated and/or analyzed during this study will be made available by the corresponding author upon reasonable request.

\section{Competing interests}

None of the authors have any competing interests to report.

\section{Funding:}

The organization had no role in the study design, in the collection, analysis or interpretation of data, in the writing of the manuscript, or in the decision to submit the manuscript for publication.

\section{Authors' contributions}

K.A. and R.O. designed the study. K.A., Rion Ozawa, Y.N., R.A., H.M., K.U., K.K., T.I.

and R.O. collected the data.

K.A., Y.N., and H.M. analyzed the data. K.A. and R.O. wrote the manuscript.

R.A., H.M., K.U., K.K., T.I. and R.O. reviewed the manuscript.

\section{Acknowledgment}

Not applicable.

\section{References}

1. Cheung, C. M. G. et al. Pachychoroid disease. Eye (Lond), 33, 14-33 https://doi.org/10.1038/s41433018-0158-4 (2019).

2. Yamashiro, K., Hosoda, Y., Miyake, M., Ooto, S. \& Tsujikawa, A. Characteristics of Pachychoroid Diseases and Age-Related Macular Degeneration: Multimodal Imaging and Genetic Backgrounds. Journal of clinical medicine, 9, https://doi.org/10.3390/jcm9072034 (2020).

3. Yanagi, Y. Pachychoroid disease: a new perspective on exudative maculopathy. Japanese journal of ophthalmology, 64, 323-337 https://doi.org/10.1007/s10384-020-00740-5 (2020).

4. Margolis, R. \& Spaide, R. F. A pilot study of enhanced depth imaging optical coherence tomography of the choroid in normal eyes. American journal of ophthalmology, 147, 811-815 https://doi.org/10.1016/j.ajo.2008.12.008 (2009).

5. Warrow, D. J., Hoang, Q. V. \& Freund, K. B. Pachychoroid pigment epitheliopathy. Retina (Philadelphia, Pa.), 33, 1659-1672 https://doi.org/10.1097/IAE.0b013e3182953df4 (2013).

6. Fung, A. T., Yannuzzi, L. A. \& Freund, K. B. Type 1 (sub-retinal pigment epithelial) neovascularization in central serous chorioretinopathy masquerading as neovascular age-related macular degeneration. Retina (Philadelphia, Pa.), 32, 1829-1837 https://doi.org/10.1097/IAE.0b013e3182680a66 (2012). 
7. Pang, C. E. \& Freund, K. B. Pachychoroid neovasculopathy. Retina (Philadelphia, Pa.), 35, 1-9 https://doi.org/10.1097/iae.0000000000000331 (2015).

8. Takahashi, A. et al. Pachychoroid Geographic Atrophy: Clinical and Genetic Characteristics. Ophthalmology. Retina, 2, 295-305 https://doi.org/10.1016/j.oret.2017.08.016 (2018).

9. Imamura, Y., Fujiwara, T., Margolis, R. \& Spaide, R. F. Enhanced depth imaging optical coherence tomography of the choroid in central serous chorioretinopathy. Retina (Philadelphia, Pa.), 29, 14691473 https://doi.org/10.1097/IAE.0b013e3181be0a83 (2009).

10. Lee, W. K. et al. Retina (Philadelphia, Pa.), 36 Suppl (1), S73-s82 https://doi.org/10.1097/iae.0000000000001346 (2016).

11. Pang, C. E. \& Freund, K. B. Pachychoroid pigment epitheliopathy may masquerade as acute retinal pigment epitheliitis. Investigative ophthalmology \& visual science, 55, 5252 https://doi.org/10.1167/iovs.14-14959 (2014).

12. Kim, J. H., Chang, Y. S., Lee, T. G. \& Kim, C. G. Choroidal vascular hyperpermeability and punctate hyperfluorescent spot in choroidal neovascularization. Investigative ophthalmology \& visual science, 56, 1909-1915 https://doi.org/10.1167/iovs.14-16000 (2015).

13. Miyake, M. et al. Choroidal neovascularization in eyes with choroidal vascular hyperpermeability. Investigative ophthalmology \& visual science, 55, 3223-3230 https://doi.org/10.1167/iovs.14-14059 (2014).

14. Miyake, M. et al. Pachychoroid neovasculopathy and age-related macular degeneration. Sci Rep, 5, 16204 https://doi.org/10.1038/srep16204 (2015).

15. Laiginhas, R., Yang, J., Rosenfeld, P. J. \& Falcão, M. Nonexudative Macular Neovascularization - A Systematic Review of Prevalence, Natural History, and Recent Insights from OCT Angiography. Ophthalmology. Retina, 4, 651-661 https://doi.org/10.1016/j.oret.2020.02.016 (2020).

16. Chen, L. et al. Nonexudative Macular Neovascularization Supporting Outer Retina in Age-Related Macular Degeneration: A Clinicopathologic Correlation., 127, 931-947 https://doi.org/10.1016/j.ophtha.2020.01.040 (2020).

17. Carnevali, A. et al. OCT Angiography of Treatment-Naïve Quiescent Choroidal Neovascularization in Pachychoroid Neovasculopathy. Ophthalmology. Retina, 1, 328-332 https://doi.org/10.1016/j.oret.2017.01.003 (2017).

18. Yanagi, Y. et al. Prevalence and Risk Factors for Nonexudative Neovascularization in Fellow Eyes of Patients With Unilateral Age-Related Macular Degeneration and Polypoidal Choroidal Vasculopathy. Investigative ophthalmology \& visual science, 58, 3488-3495 https://doi.org/10.1167/iovs.16-21167 (2017).

19. Savastano, M. C., Rispoli, M., Lumbroso, B., THE INCIDENCE OF \& NEOVASCULARIZATION IN CENTRAL SEROUS CHORIORETINOPATHY BY OPTICAL COHERENCE TOMOGRAPHY ANGIOGRAPHY. Retina (Philadelphia, Pa.), 41, 302-308 https://doi.org/10.1097/iae.0000000000002810 (2021). 
20. Yanagi, Y. et al. Incidence of Fellow Eye Involvement in Patients With Unilateral Exudative AgeRelated Macular Degeneration. JAMA ophthalmology, 136, 905-911 https://doi.org/10.1001/jamaophthalmol.2018.2154 (2018).

21. Sulzbacher, F., Schütze, C., Burgmüller, M., Vécsei-Marlovits, P. V. \& Weingessel, B. Clinical evaluation of neovascular and non-neovascular chronic central serous chorioretinopathy (CSC) diagnosed by swept source optical coherence tomography angiography (SS OCTA). Graefes Arch Clin Exp Ophthalmol, 257, 1581-1590 https://doi.org/10.1007/s00417-019-04297-z (2019).

22. Ersoz, M. G. et al. Retina (Philadelphia, Pa.), 38, 1668-1674 https://doi.org/10.1097/iae.0000000000001773 (2018).

23. Asano-Shimizu, K. et al. Early changes of vascular lesions and responses to combined photodynamic therapy in patients with polypoidal choroidal vasculopathy. International ophthalmology, 40, 1335-1345 https://doi.org/10.1007/s10792-020-01299-3 (2020).

24. Yanagi, Y. et al. Retina (Philadelphia, Pa.), 38, 1509-1517 https://doi.org/10.1097/iae.0000000000001758 (2018).

25. Sakurada, Y. et al. Retina (Philadelphia, Pa.), 38, 1977-1983 https://doi.org/10.1097/iae.0000000000002294 (2018).

\section{Figures}






\section{Figure 1}

Representative images of identifying CVH by ICGA and superimposing retinal sensitivity. The right eye of a 38-year-old man that had not been accompanied with exudative changes for more than 6 months. (a) Early phase of ICGA in an examined eye. (b) Late phase of ICGA. CVH was observed nasal superior to the fovea. (c) Identification of CVH (red line). (d) Retinal sensitivity was superimposed on c. (e) EDI-OCT image of the horizontal line scan through the fovea 


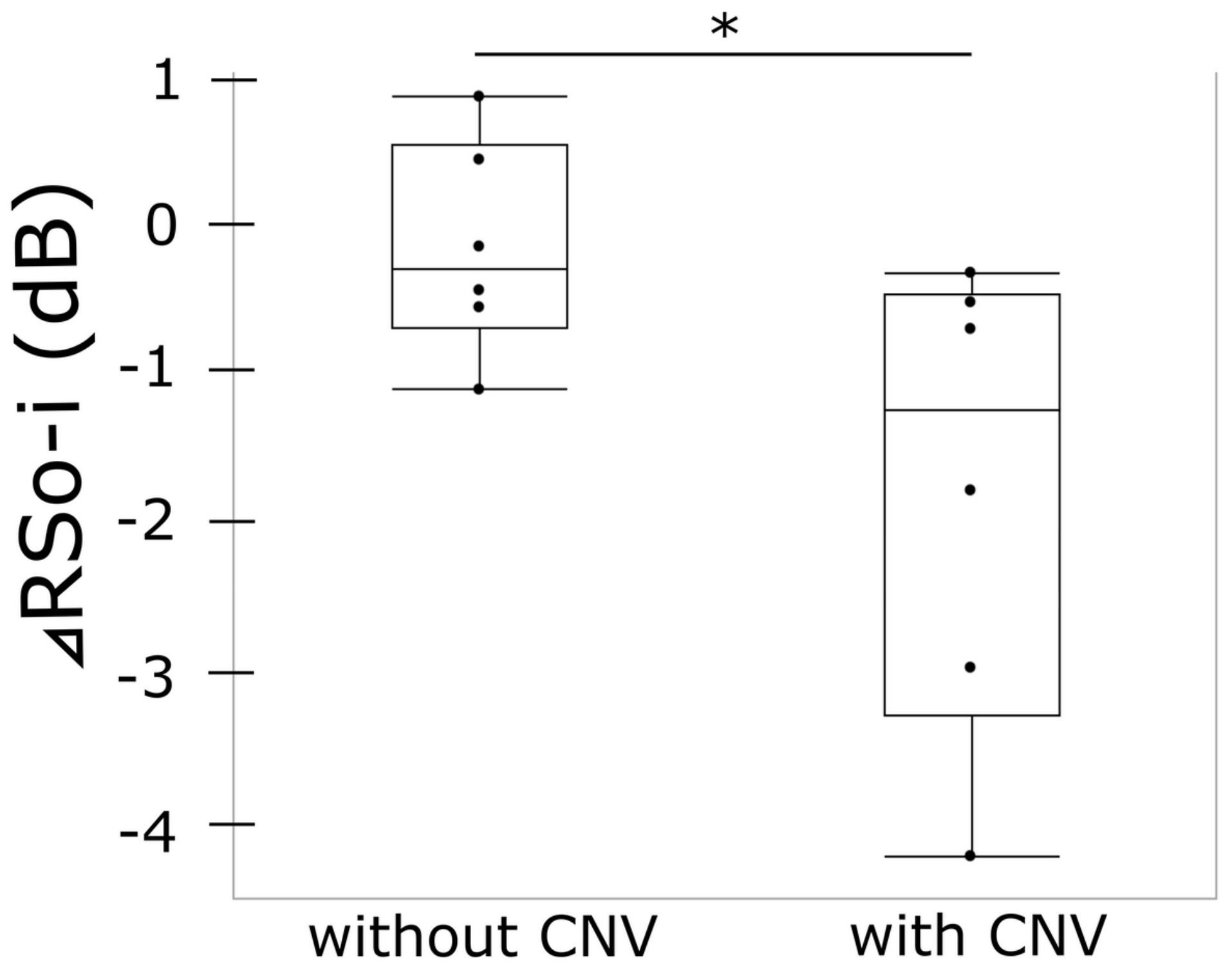

Figure 2

Difference in retinal sensitivity within and outside $\mathrm{CVH}(\triangle \mathrm{RSO}-\mathrm{i})$ in patients with $\mathrm{CNV}(\mathrm{n}=6)$ and without CNV $(n=6)$. $\triangle R S$ o-i was significantly better in eyes without CNV than in eyes with CNV $(p=0.047)$ 